\title{
Automated brain tumor segmentation and classification for MRI analysis system
}

\author{
Norhashimah Mohd Saad ${ }^{1}$, Muhamad Faizal Yaakub ${ }^{2}$, Abdul Rahim Abdullah', \\ Nor Shahirah Mohd Noor ${ }^{4}$, Nur Azmina Zainal ${ }^{5}$, Wira Hidayat Mohd Saad ${ }^{6}$ \\ 1,4,5,6 Faculty of Electronic and Computer Engineering, Universiti Teknikal Malaysia Melaka, Malaysia \\ ${ }^{2}$ Faculty of Electrical and Electronic Engineering Technology, Universiti Teknikal Malaysia Melaka, Malaysia \\ ${ }^{3}$ Faculty of Electrical Engineering, Universiti Teknikal Malaysia Melaka, Malaysia
}

\begin{tabular}{|c|c|}
\hline Article Info & ABSTRACT \\
\hline Article history: & This paper proposed a new analysis technique of brain tumor segmentation \\
\hline Received Dec 21, 2018 & and classification for Fluid Attenuated Inversion Recovery (FLAIR) \\
\hline Revised Mar 10, 2019 & Magnetic Resonance Images (MRI). 25 FLAIR MRI images were collected \\
\hline Accented Anr 252019 & from online database of Multimodal Brain Tumor Segmentation Challenge \\
\hline Ассертеd Арг 25, 2019 & $\begin{array}{l}2015 \text { (BRaTS'15). The analysis comprised four stages which are } \\
\text { preprocessing, segmentation, feature extraction and classification. Fuzzy C- }\end{array}$ \\
\hline Keywords: & $\begin{array}{l}\text { Means (FCM) was proposed for brain tumor segmentation. Mean, median, } \\
\text { mode, standard deviation, area and perimeter were calculated and utilized as }\end{array}$ \\
\hline Classification & the features to be fed into a rule-based classifier. The segmentation \\
\hline Fuzzy C-Means & formances were assessed based on Jaccard, Dice, False Positive \\
\hline MRI & similarity indices which were 0.74 and 0.83 for Jaccard and Dice indices, \\
\hline Segmentation & respectively. The technique can possibly provide high accuracy and has the \\
\hline Tumor & potential to detect and classify brain tumor from FLAIR MRI database. \\
\hline
\end{tabular}

\section{Corresponding Author:}

Norhashimah Mohd Saad,

Faculty of Electronic and Computer Engineering,

Universiti Teknikal Malaysia Melaka,

Jalan Hang Tuah Jaya, 76100 Durian Tunggal, Melaka, Malaysia.

Email: norhashimah@utem.edu.my

\section{INTRODUCTION}

Brain tumor is one of the main leading diseases which causes human death. It has become the second most important cause of death related to children and young adults [1]. Central Brain Tumor Registry of the United States (CBTRUS) reported 64,530 cases of primary brain and central nervous system tumors at the end of 2011 [1]. This brain tumor is known as a mass that is formed by the accumulation of abnormal cells [2] that grow out of control. Magnetic Resonance Imaging (MRI) is primarily applied in clinical practice in scanning and examining brain tumor. Various types of modalities use MRI to diagnose the brain tumor. The function of these modalities is to produce high level of contrast for the brain image. Furthermore, it also enhances the image quality and data gained. Fluid Attenuated Inversion Recovery (FLAIR) is the modality used for brain tumor examination. It provides better visualization in edema and tumor as it can detect contrastive elements and enhance the effect of the particularly high contrast between background tissue [3].

Although MRI is widely used for diagnosis of the brain tumor, the MRI image also provides large variance and very complex information to the size, location and the intensity of the brain tumor [1]. Due to that, the process of analyzing brain tumor is very difficult. It can only be performed by professional radiologists for the examination, analyses and final conclusion. It is time consuming and may leads to errors. 
Computer aided diagnosis (CAD) can be used as a second opinion that helps radiologists to detect any suspicious lesion in the brain scan imaging. Radiologists can finally find the conclusion based on the findings. Manual segmentation is very tedious and takes longer time [4]. Segmentation software such as ITKSNAP, 3D-Slicer and MRIcron is commonly used to help radiologist in extracting the ROI of the brain image [5-7]. These soft wares which also known as semi-automatic segmentation help the experts to analyze and evaluate the brain images [8]. Although semi-automatic segmentation techniques can give faster result, but it also comes into being different results from different experts or the same user at different times [9]. Therefore, automated segmentation integrated with the sophisticated graphical user interface (GUI) is helpful to delineate the tumor lesion.

Abdel-maksoud et al. (2015) stated that image segmentation is considered as the most essential and crucial process for facilitating the delineation, characterization, and visualization of ROI in any medical image [10]. A hybrid clustering technique was proposed by the researchers using k-Means and FCM algorithm using T1, T2 and proton density images. The objective of doing hybridization is to reduce the number of iterations resulted with less execution time. Thresholding and level set method were integrated to provide an accurate brain tumor detection. The results show that the k-Means obtained 92.49\% and FCM obtain $95.23 \%$ accuracy.

Telrandhe et al (2016) proposed k-Means clustering and Support Vector Machine (SVM) for classification of brain tumor from benign and malignant using MRI images [11]. The image was preprocessed with median filter, morphological operation and wavelet transform before segmentation of the brain tumor using k-Means clustering. Histogram Oriented Gradient (HOG) was applied to extract the ROI as the classifier input of SVM. The percentage accuracy obtained from the proposed technique is $80 \%$. Kiranmayee et al. (2017) proposed an effective analysis for MRI brain tumor using hybrid data mining techniques [12]. In the proposed approach, segmentation and classification techniques were applied using Nearest Neighbor with Generalization (NNge), Best-First Decision Tree (BFTree), LADTree, and Random Forest classifiers. These techniques were able to classify the type of abnormal and normal region include tumor region from the brain dataset. The accuracy for NNge, BFTree, decision tree, LADtree and Random Forest is $96.3 \%, 66.7 \%, 66.7 \%, 85.2 \%$ and $96.3 \%$, respectively.

Havaei et al. (2016) proposed Convolutional Neural Network (CNN) that was able to segment 75 patients with brain tumor using T1, T2 and FLAIR images [13]. The results gain from the proposed CNN technique were able to distinguish the boundaries between tumor sub-class and improve the Dice index on all tumor regions. However, the drawback of this technique is it consumed high processing time. Therefore, high performance GPU was mandatory. Based on the proposed technique, the dice coefficient, sensitivity and specificity obtain is $0.84,0.88$ and 0.84 , respectively.

El-Melegy et al. (2018) proposed an automatic brain tumor segmentation from machine learning classifier including Random Forest, Linear Discriminant Analysis (LDA) and SVM using MRI modality from T1, T2 and FLAIR images [14]. The researcher segments the whole tumor, tumor core and active core by involving the processing, feature extraction training and classification stage. The proposed technique shows that the Dice score obtained was in the range 0.8 with the highest score is from the Random Forest Classifier with 0.89. Batra and Kaushik (2017) proposed an automatic segmentation and classification technique to detect the Gliomas in the MRI brain tumor [15]. The segmentation technique proposed involved the FCM technique and the classification technique involve the SVM technique. The MRI brain tumor was segmented and the lesion was categorized into two types which are malignant and benign. SVM classifier was chosen to classify the tumor. Based on the proposed technique, the results for the automatic segmentation and classification were $98.18 \%, 97.5 \%$ and $100 \%$ for the accuracy, sensitivity and specificity, respectively.

Gurusamy (2017) proposed MRI brain tumor classification using four types of machine learning classifiers which were analyzed using Neural Network, k-NN, Naïve Bayes and SVM [17]. The segmentation technique proposed was the k-Means clustering technique. The results obtained for the Neural Network, kNN, Naïve Bayes and SVM was 97, 96,93.7 and 98, respectively. Gupta and Khanna (2019) developed a fast and efficient CAD system to detect tumor from brain MRI data [16]. Block based segmentation was applied to segment tumorous region. Then, mean, homogeneity, contrast, directionality and moments were extracted to be fed into classification stage. The extracted features were then classified as tumor or non-tumor using thresholding of block wise matching. Accuracy and precision values obtained through the experiments were $97.93 \%$ and $97.76 \%$ respectively.

Fuzzy C-Means (FCM) segmentation have been proposed by many researchers for wide variety of applications [18]. It has the ability in retaining more information from the original image compared to other segmentation methods [19]. FCM is an unsupervised machine learning technique by clustering that does not rely on various sorts of clusters but rely upon the predefined classes and training cases while arranging the data objects [20]. It specifies membership function from the data point that belongs to every cluster.

Indonesian J Elec Eng \& Comp Sci, Vol. 15, No. 3, September 2019 : 1337 - 1344 
This study proposes an automatic segmentation method that detects and diagnoses brain tumor lesions using FLAIR images. The analysis began by collecting the images of brain lesion from Multimodal Brain Tumor Segmentation Challenge 2015 (BRaTS'15) online database followed by preprocessing of the images. The proposed framework of analysis was based on the FCM segmentation technique. The results were evaluated using Jaccard, Dice, False Positive and False Negative Rates (FPR and FNR). The accuracy of the automatic segmentation method was evaluated according to the feature extraction and classification method to classify between brain tumor and normal brain.

\section{RESEARCH METHODOLOGY}

\subsection{Proposed Analysis Framework}

As depicted in Figure 1, the analysis framework began by collecting FLAIR MRI images of brain tumor lesion using online database provided in [21]-[23], followed by preprocessing stage. Segmentation technique was obtained to accurately delineate the region of interest (ROI) of the brain tumor lesion. Then, features extraction and classification were applied and the performance of the proposed technique was evaluated.

\subsection{Imaging Parameter}

FLAIR MRI images were gained from the Multimodal Brain Tumor Segmentation Challenge by Multimodal BRaTS'15 database online [21]. These data comprised MRI modality with MHA format in 16 bits and were converted into Neuroimaging Informatics Technology Initiative (NIFTI) format using ITKSNAP software. The format of the dataset was then changed to Digital Imaging and Communications in Medicine (DICOM) format using (X) MedCon applications. The dataset was encoded in 8-bit DICOM to standardize each image for the proposed segmentation method. The parameter of the dataset was $240 \times 240$ of pixel resolution with 25 slices of each patient. Each dataset was provided with reference image to compare with the proposed segmentation method of the ROI for brain tumor lesion.

\subsection{Preprocessing}

The preprocessing stage was performed to improve the brain tumor images. Normalization intensity was applied to normalize the pixel intensity value. The actual MRI image that contained 16-bit depth pixel intensity was normalized and was down-sampling into 8-bit depth pixel intensity value to standardize the input image with the MRI dataset. The image normalization with its histogram is as shown in Figure 2.

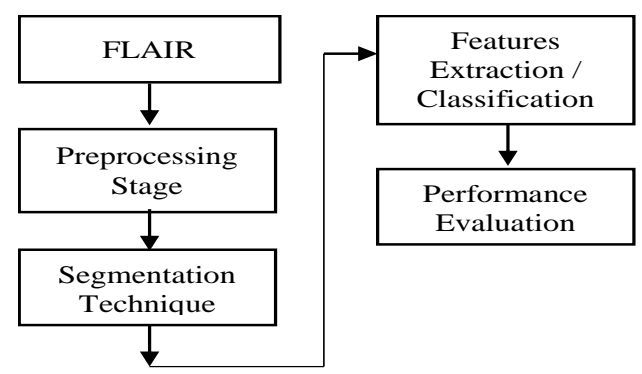

Figure 1. Flowchart of the Analysis
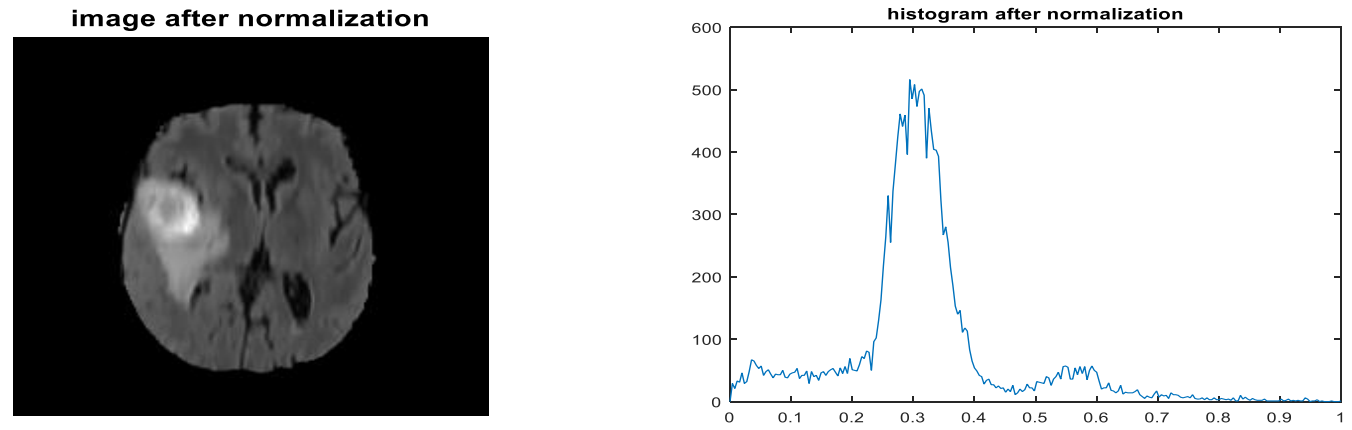

Figure 2. Image after normalization with its histogram 


\subsection{Segmentation}

FCM was the clustering method performed by grouping or portioning the information pattern with respect to the desired information pattern group [24]. Each data point in the data clustering belonged to the degree of certain cluster by a membership value. FCM method consisted of $n$ vectors collection which was classified through the fuzzy group of $\mathrm{c}$ to find the cluster center in every group. The fuzzy portioning was applied to a data point given in the membership values which could specify the values between zero and from the cluster degree of belonging. The summarized step of FCM framework is as follows [25]:

Step 1: Select the image after the preprocessing process

Step 2: Convert the image format to double to enhance the range of the pixel

Step 3: Identify the number of the iteration

Step 4: Obtain the size of the image

Step 5: Repeat the structure by calculating the possible distance

Step 6: Calculate the possible distance by repeating the matrix for generating the large item

Step 7: Identify large component of data of the pixel by starting the iteration

Step 8: Stop the iteration if the possible identification elapses

Step 9: Segmentation is generated

The membership matrix $U$ is initialized with intensity cluster values from 0 to 1 . The algorithm is based on the minimization of the objective function, as shown in (1) [25].

$$
J_{m}=\sum_{i=1}^{N} \sum_{j=1}^{C} u_{i j}^{m}\left\|x_{i}-c_{j}\right\|^{2}, 1 \leq m<0
$$

where:

$\mathrm{m}=$ real number $\geq 1$,

$u_{i j}=$ membership degree of $x_{i}$ in the cluster $\mathrm{j}$,

$x_{i}=i$ of d-dimensional measured data,

$c_{j}=\mathrm{d}$-dimension center of the cluster,

$\|*\|=$ any norm expressing the similarity between any measured data and the center.

Fuzzy partitioning of the objective function was carried out through an iterative optimization shown above, with the update of membership $u_{i j}$ and the cluster centers $c_{j}$ by:

$$
u_{i j}=\frac{1}{\sum_{k=1}^{c}\left(\frac{\left\|x_{i}-c_{j}\right\|}{\left\|x_{i}-c_{k}\right\|}\right)^{\frac{2}{m-1}}}, c_{j} \frac{\sum_{i=1}^{N} u_{i j}^{m} \cdot x_{i}}{\sum_{i=1}^{N} u_{i j}^{m}}
$$

When $\max _{i j}\left\{\left|u_{i j}{ }^{(k+1)}-u_{i j}{ }^{(k)}\right|\right\}<\varepsilon$, the iteration will stop, where $\varepsilon$ is the termination criterion between 0 and 1 , and $k$ is the iteration steps.

This procedure converged to a local minimum or a saddle point of $J_{m}$. The algorithm of the following steps is described below:

1. $U=\left[u_{i j}\right]$ matrix,$u^{(0)}$ is initialized with the random values between zero and one.

2. Calculate the cluster of center, using (3).

$c_{j} \frac{\sum_{i=1}^{N} u_{i j}^{m} \cdot x_{i}}{\sum_{i=1}^{N} u_{i j}^{m}}$

3. $U^{(k)}, U^{(k+1)}$ is updated.

4. Calculate the table of the fuzzy membership function, using (4).

$$
u_{i j}=\frac{1}{\sum_{k=1}^{c}\left(\frac{\left\|x_{i}-c_{j}\right\|}{\left\|x_{i}-c_{k}\right\|}\right)^{\frac{2}{m-1}}}
$$

5. Stop when $\left\|U^{(k+1)}-U^{(k)}\right\|<\varepsilon$; otherwise return to step 2 .

The binary image and threshold level were obtained from FCM method by the matlab command fcmthresh for FCM. The change for the cut-off position was 0 until 1 to indicate the cut between the small and middle classes and the middle and large classes. 


\subsection{Feature Extraction}

Feature extraction was applied to represent the characteristics of the ROI which were obtained from the segmentation results using statistical data. The statistical data were calculated using the formulas of mean, median, mode, standard deviation, area and perimeter of the ROI. Each value of the statistical data was to determine the location, shape and other elements in the lesions [26].

$$
\begin{aligned}
& \mu=\frac{1}{N} \sum_{i=1}^{N=1} P(i) \\
& \sigma=\sqrt{\frac{1}{N}} \sum_{i=1}^{N=1}(P(i)-\mu) \\
& \text { Median }=\frac{1}{2}(n+1) \text { value } \\
& \text { Perimeter }=\text { Length }(\text { boundary })
\end{aligned}
$$

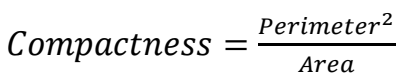

\section{RESULTS AND ANALYSIS}

\subsection{Segmentation Results}

Table 1 shows the results of brain tumor with FCM segmentation compared with the manual reference image given in the dataset. The obtained segment is compared with the extracted manual segment in terms of its similarity and the tumor area. As presented in Table 1, the obtained FCM segmentation results have been quite satisfactory. However, due to the existence of noise, FCM does not consider the spatial information which makes it sensitive to the noise. The table portrays the FLAIR, manual reference and FCM results for tumor segmentation.

Table 1. The Segmentation Results of the Brain Tumor Lesions

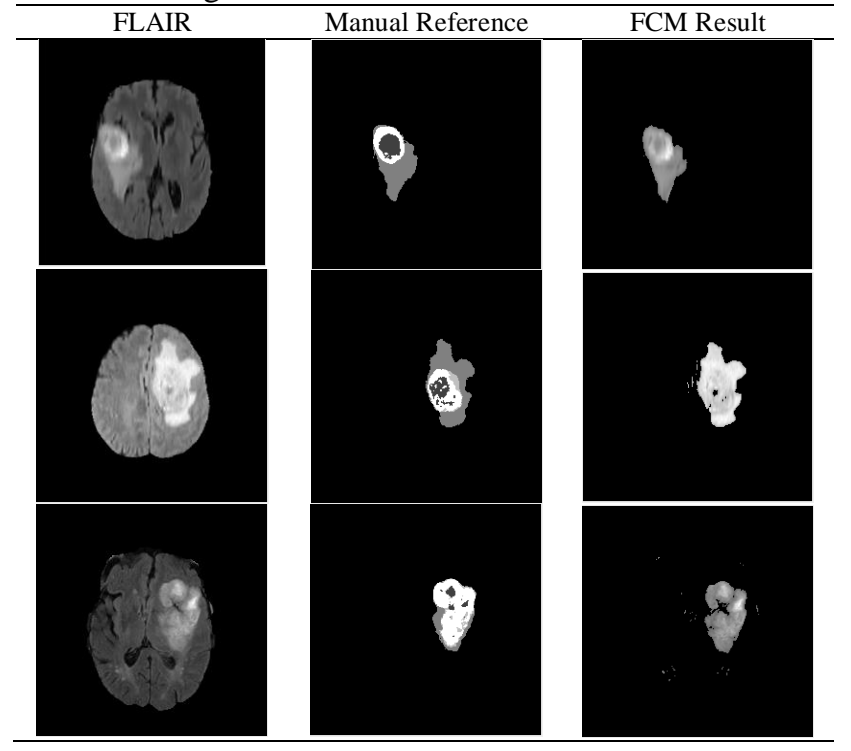

Figure 3 shows the performance results of Jaccard index for the brain tumor lesions. Only patient 10 showed samples of Jaccard index below 0.5 with the index of 0.43 . The highest index of the Jaccard index was in patient 5 with the reading of 0.93 . Figure 4 shows the results of the Dice index. All of the patients obtained Dice index above 0.5 with the lowest value of 0.6 in patient 10 . The highest sample of the Dice index was 0.96 in patient 5. Figure 5 shows the result of the FPR performance, that measures the index of oversegmentation error. Only patient 10 failed to obtain error rate below 0.5 with the value of 0.56 . The lowest error was obtained in patient 4, 13, 14, 17 and 20 with the FPR reading of 0 . Figure 6 shows the readings of the FNR performance for undersegmentation error. All of the patient samples obtained 
best results which were below 0.5 with the highest reading at 0.41 and the lowest reading at 0.001 from patient 10.

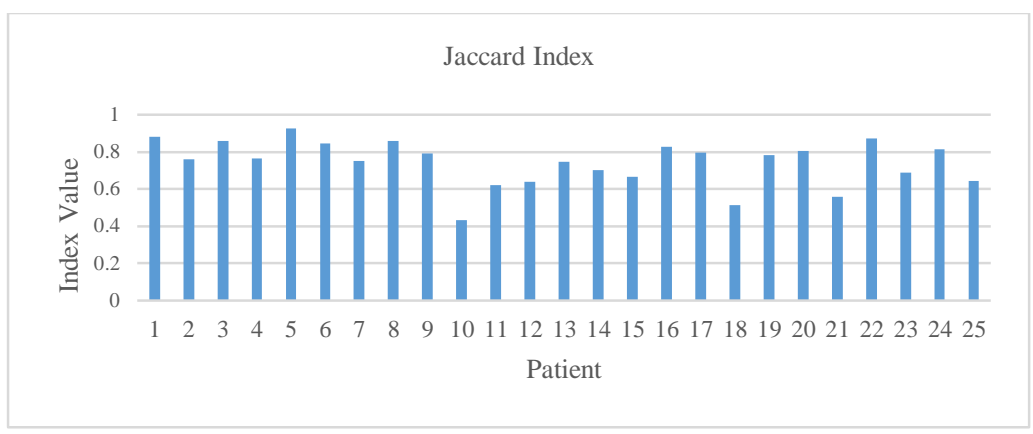

Figure 3. Jaccard Index Results for FCM Segmentation

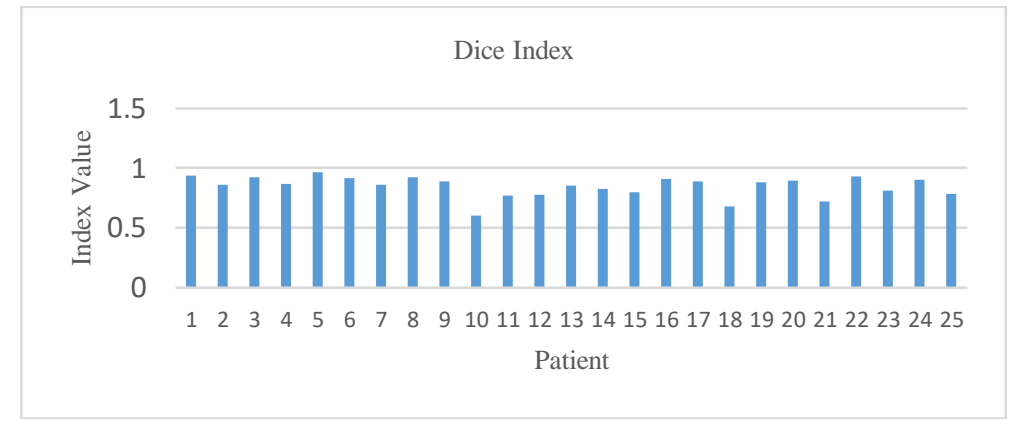

Figure 4. Dice Index Results for FCM Segmentation

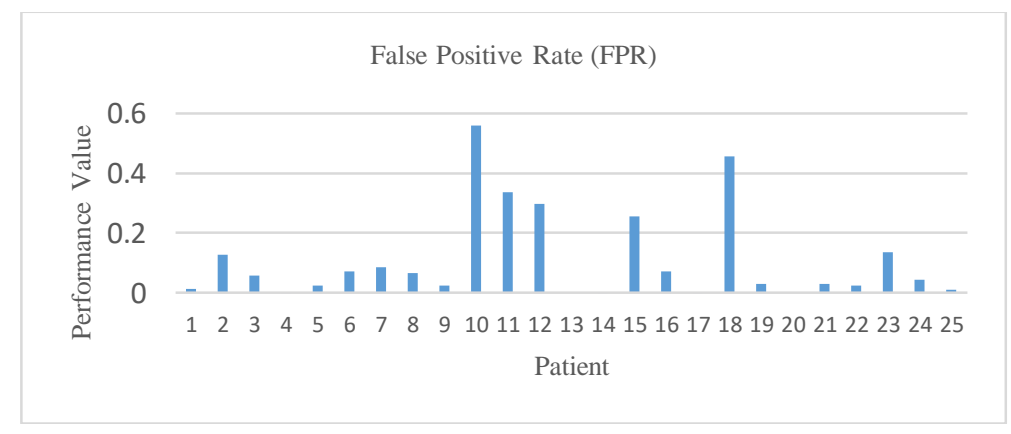

Figure 5. FPR Results for FCM Segmentation

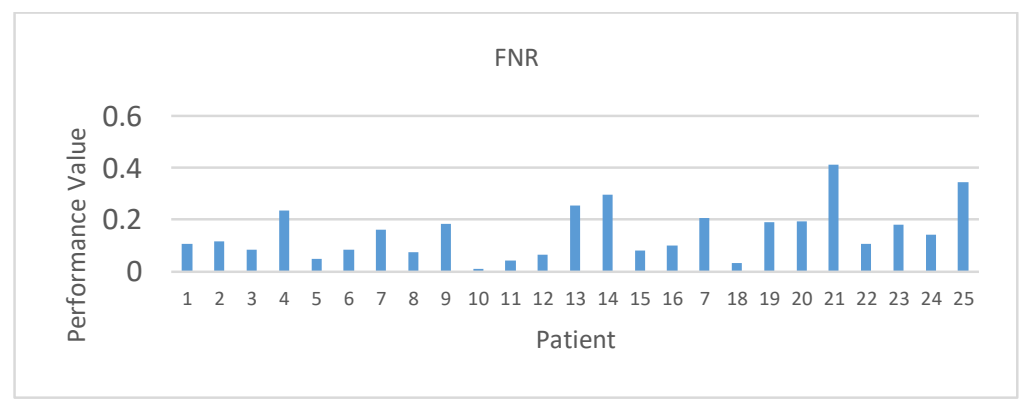

Figure 6. FNR Results for FCM Segmentation 


\subsection{Feature Extraction and Classification Results}

Table 2 shows the feature extraction using mean, compactness, median and boundary, explained in Section 2. For each feature, minimum and maximum values corresponding to all features were identified for tumor and normal images. The range obtained for each feature produced a threshold value respective to it. The classification of the brain is tumor if the feature values lie in the threshold range of brain tumor MRI, else the MRI is normal.

Table 2. The Feature Extracted from the Brain Area

\begin{tabular}{ccccc}
\hline Symbol & \multicolumn{2}{c}{ Brain Tumor } & \multicolumn{2}{c}{ Normal Brain } \\
\hline Range & Min & Max & Min & Max \\
Mean & 0.34 & 0.87 & 0.12 & 0.39 \\
Compactness & 13 & 433 & 14.5 & 18.9 \\
Median & 0.33 & 0.95 & 0.13 & 0.56 \\
Boundary & 0.2 & 0.75 & 0.09 & 0.37 \\
\hline
\end{tabular}

The accuracy of the brain tumor segmentation was $96 \%$ over 25 samples of image. This is due to the Patient 25 has obtained below than 0.5 in the mean value. Supposedly the ROI brain tumor lesions must provide higher intensity value while normal brain must provide lower intensity value.

\section{CONCLUSION}

In this study, the FCM segmentation method is implemented for the segmentation of brain tumor using FLAIR images. The segmentation technique with the manual reference from the same database is compared to verify the accuracy. The results for the Jaccard index, Dice index, FPR and FNR are 0.74, 0.83, 0.11 and 0.14 , respectively. Based on these results, FCM gives better segmentation result for the brain tumor based on the high values of Jaccard index and Dice index and low values of FPR and FNR. The segmentation method proposed can segment the brain tumor lesions in 25 samples of patients and it is also suitable for the analysis and classification.

\section{ACKNOWLEDGEMENTS}

The authors would like to thank Universiti Teknikal Malaysia Melaka and Ministry of Higher Education for supporting this research under PJP/2017/FTK/HI14/S01546.

\section{REFERENCES}

[1] Pushpa Rathi, V. (2012). "Brain Tumor MRI Image Classification with Feature Selection and Extraction using Linear Discriminant Analysis". International Journal of Information Sciences and Techniques, 2(4), pp.131-146.

[2] Roy, S., \& Bandyopadhyay, S. K. (2012). "Detection and Quantification of Brain Tumor from MRI of Brain and it's Symmetric Analysis". International Journal of Information and Communication Technology Research, 2(6), pp.477-483.

[3] Drevelegas, A. (2010). "Imaging Modalities in Brain Tumors". In Imaging of Brain Tumors with Histological Correlations, pp.13-33.

[4] Gordillo, N., Montseny, E. and Sobrevilla, P. (2013). "State of the Art Survey on MRI Brain Tumor Segmentation. Magnetic Resonance Imaging, 31(8), pp.1426-1438.

[5] Maltbie, E., Bhatt, K., Paniagua, B., Smith, R.G., Graves, M.M., Mosconi, M.W., Peterson, S., White, S., Blocher, J., El-Sayed, M. and Hazlett, H.C. (2012). "Asymmetric Bias in User Guided Segmentations of Brain Structures". Neuroimage, 59(2), pp.1315-1323.

[6] Fedorov, A., Beichel, R., Kalpathy-Cramer, J., Finet, J., Fillion-Robin, J.C., Pujol, S., Bauer, C.,Jennings, D., Fennessy, F., Sonka, M. and Buatti, J. (2012). "3D Slicer as an Image Computing Platform for The Quantitative Imaging Network”. Magnetic Resonance Imaging, 30(9), pp.1323-1341.

[7] Delon-Martin, C., Plailly, J., Fonlupt, P., Veyrac, A. and Royet, J.P. (2013). "Perfumers' Expertise Induces Structural Reorganization in Olfactory Brain Regions". Neuroimage, 68, pp.55-62.

[8] Sharma, N. and Aggarwal, L.M. (2010). "Automated Medical Image Segmentation Techniques". Journal of Medical Physics, 35(1), pp.3-14.

[9] Liu, J., Li, M., Wang, J., Wu, F., Liu, T. and Pan, Y. (2014). "A Survey of Mri-Based Brain Tumor Segmentation Methods". Tsinghua Science and Technology, 19(6), pp.578-595.

[10] Abdel-Maksoud, E., Elmogy, M. and Al-Awadi, R. (2015). "Brain Tumor Segmentation Based on a Hybrid Clustering Technique”. Egyptian Informatics Journal, 16(1), pp.71-81. 
[11] Telrandhe, S.R., Pimpalkar, A. and Kendhe, A. (2016). "Implementation of Brain Tumor Detection Using Segmentation Algorithm and SVM". International Journal on Computer Science and Engineering, 8(7), pp. 278-284.

[12] Kiranmayee, B.V., Rajinikanth, T.V. and Nagini, S. (2017). "Effective Analysis of Brain Tumor Using Hybrid Data Mining Techniques". International Journal of Advanced Research in Computer Science, 8(7), pp. 286-293.

[13] Havaei, M., Davy, A., Warde-Farley, D., Biard, A., Courville, A., Bengio, Y., Pal, C., Jodoin, P.M. and Larochelle, H. (2017). "Brain Tumor Segmentation with Deep Neural Networks". Medical Image Analysis, 35, pp.18-31.

[14] El-Melegy, M.T., El-Magd, K.M.A., Ali, S.A., Hussain, K.F. and Mahdy, Y.B. (2018). "A Comparative Study of Classification Methods for Automatic Multimodal Brain Tumor Segmentation". In Innovative Trends in Computer Engineering (ITCE), 2018 International Conference on Innovative Trends in Computer Engineering (ITCE 2018), pp. 36-41.

[15] Batra, A. and Kaushik, G. (2017). "SECTUBIM: Automatic Segmentation and Classification of Tumeric Brain MRI Images using FHS (FCM, HWT and SVM)”. International Journal of Engineering Science, 7(6), pp. 13190-13194.

[16] Gurusamy, R. and Subramaniam, V. (2017). “A Machine Learning Approach for MRI Brain Tumor Classification”. Computers, Materials \& Continua, 53(2), pp.91-108.

[17] Gupta, N. and Khanna, P. (2015). "A Fast and Efficient Computer Aided Diagnostic System to Detect Tumor from Brain Magnetic Resonance Imaging”, International Journal of Imaging Systems and Technology, 25 (2), pp. 0899-9457.

[18] Kannan, S. R., Pandiyarajan, R., \& Ramathilagam, S. (2010). "Effective Weighted Bias Fuzzy C-Means in Segmentation of Brain MRI. 2010 Int. Conf. of IEEE Intelligent and Advanced Systems (ICIAS), pp.1-6.

[19] Z. Yang, F. Chang and W. Shitong, (2009). "Robust Fuzzy Clustering-Based Image Segmentation". Applied Soft Computing, 9(1), pp.80-84.

[20] Malathi R., \& Nadirabanu Kamal A. R. (2015). "Brain Tumor Detection and Identification Using K-Means Clustering Technique". Proceedings of the UGC Sponsored National Conference on Advanced Networking and Applications, pp.14-18.

[21] Multimodal Brain Tumor Segmentation Challenge 2018 (BRATS). [online]. http://braintumorsegmentation.org/.

[22] Kistler et. al. (2013). "The Virtual Skeleton Database: An Open Access Repository for Biomedical Research and Collaboration”. Journal of Medical Internet Research, 15(11), e245, pp.1-14.

[23] B. H. Menze et al. (2015). "The Multimodal Brain Tumor Image Segmentation Benchmark (BRATS)", in IEEE Transactions on Medical Imaging, vol. 34, no. 10, pp. 1993-2024.

[24] Muda, A. F., Saad, N. M., Abu Bakar, S. A. R., Muda, S., \& Abdullah, A. R. (2015). "Integration of Fuzzy CMeans with Correlation Template and Active Contour for Brain Lesion Segmentation in Diffusion-Weighted MRI". ARPN Journal of Engineering and Applied Sciences, Vol. 10, no. 3, pp. 1138-1144.

[25] Bilenia A., Sharma D., Raj H., Raman R., Bhattacharya M. "(2019) Brain Tumor Segmentation with Skull Stripping and Modified Fuzzy C-Means”. In: Satapathy S., Joshi A. (eds) Information and Communication Technology for Intelligent Systems. Smart Innovation, Systems and Technologies, vol. 106. Springer, Singapore.

[26] N.M. Saad, Syed A.R. Abu-Bakar, A.F. Muda, Sobri Muda, A. R. Syafeeza, (2014), International Journal of Engineering and Technology (IJET), 6 (6), pp.2685-2697. Engg Journals Publications.

[27] N. Mohd Saad, S.A.R. Abu-Bakar, Sobri Muda, M. Mokji, A.R. Abdullah, (2012). "Fully Automated Region Growing Segmentation of Brain Lesion in Diffusion-weighted MRI". IAENG International Journal of Computer Science, 39(2), pp. 155-164. 